\title{
Resonant Magnetic Vortices
}

\author{
Yves Décanini用 and Antoine Folacci间 \\ SPE, UMR CNRS 6134, Equipe Physique Semi-Classique (et) de la Matière Condensée \\ Université de Corse, Faculté des Sciences, BP 52, 20250 Corte, France
}

(Dated: November 2, 2018)

\begin{abstract}
By using the complex angular momentum method, we provide a semiclassical analysis of electron scattering by a magnetic vortex of Aharonov-Bohm-type. Regge poles of the $S$-matrix are associated with surface waves orbiting around the vortex and supported by a magnetic field discontinuity. Rapid variations of sharp characteristic shapes can be observed on scattering cross sections. They correspond to quasibound states which are Breit-Wigner-type resonances associated with surface waves and which can be considered as quantum analogues of acoustic whispering-gallery modes. Such a resonant magnetic vortex could provide a new kind of artificial atom while the semiclassical approach developed here could be profitably extended in various areas of the physics of vortices.
\end{abstract}

PACS numbers: 03.65.Nk, 03.65.Sq, 73.21.-b, 47.32.Cc

\section{INTRODUCTION}

Modern electronic technology is based on the control of the current and, with this aim in view, artificial structures such as quantum wires, dots and other nanoscaled devices have been developed (see, for example, Ref. 11 and references therein). In this context, the Aharonov-Bohm $(\mathrm{AB})$ effect 2, 3, 4 is nowadays often involved: a magnetic flux threading a confined region with leads is used to modify the electron transport behavior. With this in mind, we study, in this paper, an artificial structure which presents richer scattering properties than the ordinary flux lines usually considered. Such a system could provide a new kind of artificial atom.

Here, we consider the scattering of a spinless electron (mass $M$, charge $-e$ and total energy $E$ ) by the magnetic vortex defined, in the cylindrical coordinate system $(\rho, \varphi, z)$, by the electromagnetic potential

$$
V=\left\{\begin{array}{ccc}
+\infty & \text { for } \rho<R_{1}, \\
0 & \text { for } \rho>R_{1},
\end{array}, \mathbf{A}=\left\{\begin{array}{cc}
\frac{\Phi}{2 \pi \rho} \mathbf{e}_{\varphi} & \text { for } \rho<R_{2} \\
0 & \text { for } \rho>R_{2}
\end{array}\right.\right.
$$

with $R_{1}<R_{2}$. In Eq. (11), the scalar potential describes a hard core which prevents the electron from entering the region $\rho<R_{1}$ while the vector potential describes, in the radial gauge, the magnetic field $\mathbf{B}=(\Phi / 2 \pi \rho)\left(\delta(\rho)-\delta\left(\rho-R_{2}\right)\right) \mathbf{e}_{\mathbf{z}}$. This field consists of a flux line at $\rho=0$ (the usual $\mathrm{AB}$ flux line) and an infinitely thin magnetic field shell localized at $\rho=R_{2}$. Inside the region $\rho<R_{2}$, the total flux is given by $\Phi$ while outside this region it vanishes. Such a vortex was first suggested by Aharonov in order to avoid the ambiguities of the incoming wave function of the electron in the $\mathrm{AB}$ scattering, ambiguities arising from the long range behavior of the vector potential. It was studied by Liang in Ref. 回.

\footnotetext{
*Electronic address: decanini@univ-corse.fr

${ }^{\dagger}$ Electronic address: folacci@univ-corse.fr
}

In this article, the scattering of the electron is analyzed from a semiclassical point of view by using the complex angular momentum (CAM) method. It should be noted that this method has been extensively used in several domains of scattering theory since the pioneering work of Watson [6] dealing with the propagation and diffraction of radio waves around the earth (see the monographs of Newton [7] and of Nussenzveig [8] and references therein for various applications in quantum mechanics, nuclear physics, electromagnetism, optics, acoustics and seismology). As far as we know, the CAM method has never been introduced in the context of the $\mathrm{AB}$ effect (see, however, the end of Ref. 9 where Berry envisages the possibility of such an approach) or in order to study the electron scattering by a magnetic field as well as its resonant aspects. As we shall show below, this semiclassical approach permits us to emphasize the aspects of scattering linked to time-reversal invariance breaking as well as the rich resonant properties of the magnetic vortex and more particularly the existence of quasibound states associated with surface waves orbiting around the vortex and supported by the magnetic field shell.

\section{EXACT $S$-MATRIX AND RESONANCES}

From now on, we treat our problem in a twodimensional setting, ignoring the $\mathrm{z}$ coordinate. We denote by $\hat{H}$ the Hamiltonian of the electron and we introduce its wave number $k=\sqrt{2 M E} / \hbar$ as well as the quantum flux parameter $\alpha=-e \Phi / 2 \pi \hbar$. We are first interested by the construction of the $S$-matrix. Because of the cylindrical symmetry of the vortex, the $S$-matrix is diagonal and its elements $S_{\ell m}$ are given by $S_{\ell m}=S_{\ell} \delta_{\ell m}$. For a given angular momentum index $\ell \in \mathbf{Z}$, the coefficient $S_{\ell}$ is obtained from the partial wave $\Psi_{\ell}$ solution of the following problem [10]:

(i) $\Psi_{\ell}$ satisfies the time-independent Schrödinger equation $(\hat{H}-E) \Psi_{\ell}=0$,

(ii) $\Psi_{\ell}$ vanishes at $\rho=R_{1}$ (impenetrability of the hard 
core of radius $R_{1}$ ) while $\Psi_{\ell}$ as well as its normal derivative are both continuous at $\rho=R_{2}$ (which guarantee the continuity of the probability density and of the radial component of the probability current at $\rho=R_{2}$.5]),

(iii) at large distance, $\Psi_{\ell}$ has the asymptotic behavior

$$
\begin{aligned}
\Psi_{\ell}(\rho, \varphi) \underset{\rho \rightarrow+\infty}{\sim} & \frac{1}{\sqrt{2 \pi k \rho}}\left(e^{-i(k \rho-\ell \pi / 2-\pi / 4)}\right. \\
+ & \left.S_{\ell} e^{i(k \rho-\ell \pi / 2-\pi / 4)}\right) e^{i \ell \varphi}
\end{aligned}
$$

In the region $R_{1}<\rho<R_{2}, \hat{H}$ is the standard AB Hamiltonian given by

$$
\hat{H}=-\frac{\hbar^{2}}{2 M}\left[\frac{\partial^{2}}{\partial \rho^{2}}+\frac{1}{\rho} \frac{\partial}{\partial \rho}+\frac{1}{\rho^{2}}\left(\frac{\partial}{\partial \varphi}-i \alpha\right)^{2}\right]
$$

and the solution of (i) is expressible in terms of Bessel functions (see [11]) as a linear combination of $J_{\ell-\alpha}(k \rho) e^{i \ell \varphi}$ and $H_{\ell-\alpha}^{(1)}(k \rho) e^{i \ell \varphi}$. Let us recall that in the usual AB scattering, the Bessel function indices are $|\ell-\alpha|$ with $\ell \in \mathbf{Z}$. Here, absolute values are unnecessary because we do not require the regularity of the modes at $\rho=0$. In the region $\rho>R_{2}, \hat{H}$ reduces to the free Hamiltonian and the solution of (i) can be constructed from $J_{\ell}(k \rho) e^{i \ell \varphi}$ and $H_{\ell}^{(1)}(k \rho) e^{i \ell \varphi}$. As a consequence, the partial wave $\Psi_{\ell}$ solution of (i) and (ii) can be obtained exactly. Then, by using the standard asymptotic behavior of Hankel functions [11], we find from (iii) the expression of the diagonal elements $S_{\ell}$ of the $S$-matrix:

$$
S_{\ell}(k)=1-2 \frac{D_{\ell}^{(1)}(k)}{D_{\ell}(k)}
$$

where $D_{\ell}^{(1)}(k)$ and $D_{\ell}(k)$ are two $3 \times 3$ determinants which are explicitly given by

$$
\begin{aligned}
& D_{\ell}^{(1)}(k)=n_{\ell-\alpha}(k) J_{\ell}\left(k R_{2}\right)-d_{\ell-\alpha}(k) J_{\ell}^{\prime}\left(k R_{2}\right), \\
& D_{\ell}(k)=n_{\ell-\alpha}(k) H_{\ell}^{(1)}\left(k R_{2}\right)-d_{\ell-\alpha}(k) H_{\ell}^{(1)^{\prime}}\left(k R_{2}\right),
\end{aligned}
$$

with

$$
\begin{aligned}
& n_{\mu}(k)=H_{\mu}^{(1)}\left(k R_{1}\right) J_{\mu}^{\prime}\left(k R_{2}\right)-J_{\mu}\left(k R_{1}\right) H_{\mu}^{(1)^{\prime}}\left(k R_{2}\right),(4 \mathrm{c}) \\
& d_{\mu}(k)=H_{\mu}^{(1)}\left(k R_{1}\right) J_{\mu}\left(k R_{2}\right)-J_{\mu}\left(k R_{1}\right) H_{\mu}^{(1)}\left(k R_{2}\right) .(4 \mathrm{~d})
\end{aligned}
$$

The unitarity of the $S$-matrix [7], which expresses the probability conservation, can be easily verified by using elementary properties of Bessel functions. The reciprocity property [7], which is associated with timereversal invariance, is not satisfied because $S_{\ell}$ is not an even function of $\ell$. Furthermore, the $S$-matrix is not invariant under the change $\alpha \rightarrow \alpha+1$. Here, and by contrast to the case of the ordinary AB scattering, the electron can distinguish between two fluxes which differ by an integer multiple of $2 \pi \hbar / e$.

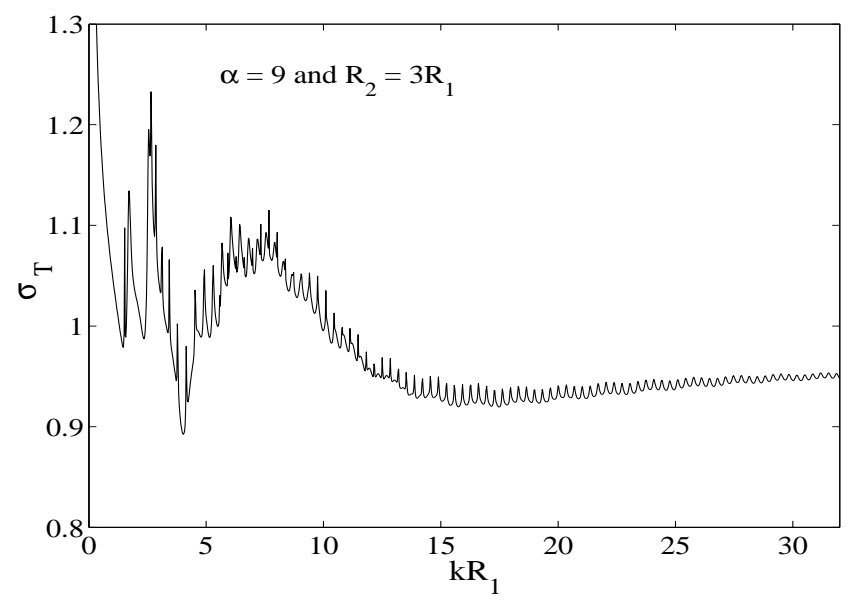

FIG. 1: The normalized total cross section $\sigma_{T}$.

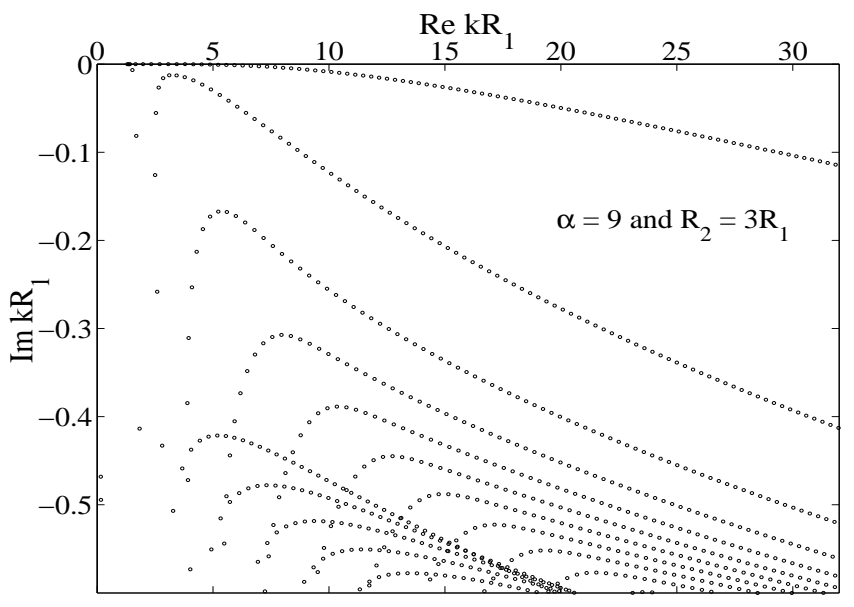

FIG. 2: Scattering resonances in the complex $k R_{1}$-plane.

From the $S$-matrix, one can construct the scattering amplitude

$$
f(k, \varphi)=\sqrt{\frac{1}{2 i \pi k}} \sum_{\ell=-\infty}^{+\infty}\left(S_{\ell}(k)-1\right) e^{i \ell \varphi}
$$

while the total scattering cross section can be obtained by using the optical theorem:

$$
\sigma_{T}(k)=\frac{1}{2 \pi} \sqrt{\frac{8 \pi}{k}} \operatorname{Im}\left(e^{-i \pi / 4} f(k, \varphi=0)\right) .
$$

In Fig. 1, we present the total cross section as a function of $k R_{1}$. As far as the numerical aspects of our work are concerned, we restrict ourselves to the particular configuration $\alpha=9$ and $R_{2}=3 R_{1}$ (we note, however, that the results emphasized numerically are very general) and we furthermore use the reduced wave number $k R_{1}$ instead of the wave number $k$. Rapid variations of sharp characteristic shapes can be observed. This strongly fluctuating behavior is due to scattering resonances. These resonances are the poles of the $S$-matrix lying in the fourth 
quadrant of the complex $k$-plane and they are determined by solving

$$
D_{\ell}(k)=0 \quad \text { for } \quad \ell \in \mathbf{Z} \text {. }
$$

The solutions of $(耳)$ are denoted by $k_{\ell p}=k_{\ell p}^{(o)}-i \Gamma_{\ell p} / 2$ where $k_{\ell p}^{(o)}>0$ and $\Gamma_{\ell p}>0$, the index $p$ permitting us to distinguish between the different roots of (7) for a given $\ell$. In the immediate neighborhood of the resonance $k_{\ell p}$, $S_{\ell}(k)$ has the Breit-Wigner form, i.e., is proportional to

$$
\frac{\Gamma_{\ell p} / 2}{k-k_{\ell p}^{(o)}+i \Gamma_{\ell p} / 2}
$$

As a consequence, when a pole of the $S$-matrix is sufficiently close to the real axis in the complex $k$-plane, it has an appreciable influence on the total cross section. In Fig. 2, resonances are exhibited. A one-to-one correspondence between the peaks of $\sigma_{T}$ in Fig. 11 and the resonances near the real $k R_{1}$-axis can be clearly observed. In the scattering of an electron with wave number $k_{\ell p}^{(o)}$, a decaying state of the electron-vortex system is formed. It has a finite lifetime proportional to $1 / \Gamma_{\ell p}$ and which increases with $\alpha$. When the corresponding scattering resonance can be observed on the total cross section, it is a long-lived state. Because of these quasibound states, the magnetic vortex behaves as a kind of artificial atom.

\section{SEMICLASSICAL ANALYSIS}

Using the CAM method, we can provide a physical picture of the scattering process in term of diffraction by surface waves and a physical explanation of the mechanism of resonance excitation valid for high wave numbers. We shall work from the scattering amplitude but we are fully aware of the fact that a more rigorous (but also more longer) analysis could be done from the trace of the Green function. We first apply the Poisson summation formula to the scattering amplitude (5). We have

$$
f(k, \varphi)=\sqrt{\frac{1}{2 i \pi k}} \sum_{m=-\infty}^{+\infty} \int_{-\infty}^{+\infty} d \lambda\left(S_{\lambda}(k)-1\right) e^{i \lambda(\varphi+m 2 \pi)}
$$

We now go over the complex $\lambda$-plane. $\lambda$ is then called the CAM and $S_{\lambda}(k)$ is then an analytical extension of $S_{\ell}(k)$ into the CAM plane. We can then deform the path of integration in (9) taking into account the possible singularities. The only singularities that are met are the poles of the S-matrix lying in the CAM plane. They are known as Regge poles [7, \&] and are determined by solving

$$
D_{\lambda}(k)=0 \quad \text { for } \quad k>0 \text {. }
$$

The solutions of $(10)$ are denoted by $\lambda_{p}$, the index $p$ permitting us to distinguish between the different roots.

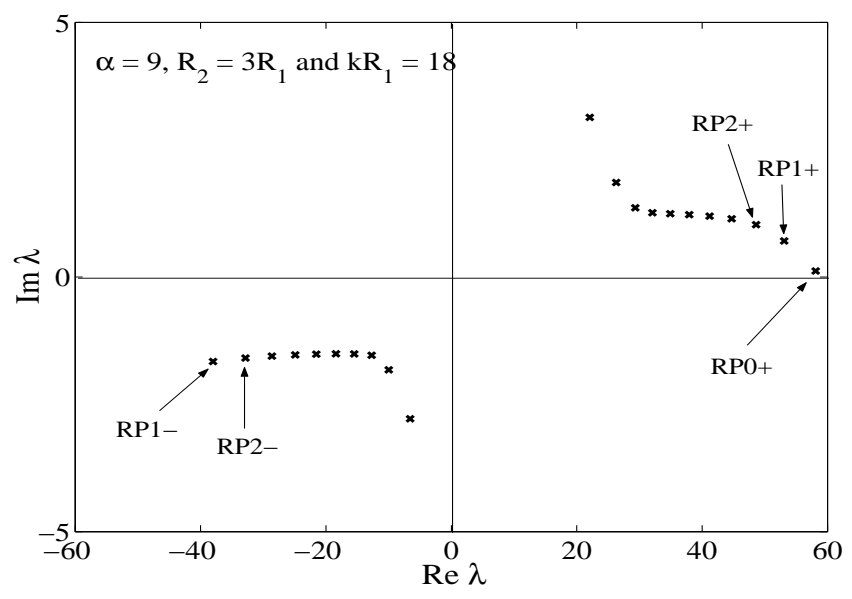

FIG. 3: Regge poles in the complex angular momentum plane.

We can then extract from (9) the contribution of a residue series over Regge poles given by

$$
f_{S}(k, \varphi)=\sqrt{\frac{2 i \pi}{k}} \sum_{p} \pm r_{p}(k) \sum_{m=1}^{+\infty} e^{i \lambda_{p}(k)(\varphi \pm m 2 \pi)}
$$

where $r_{p}(k)=$ residue $\left(S_{\lambda}(k)\right)_{\lambda=\lambda_{p}(k)}$. Here the + and signs are associated with the Regge poles lying respectively in the first and in the third quadrant of the CAM plane. It should be noted that $f$ differs from $f_{S}$ by contour integrals which provide an uniform approximation valid in a large range around $\varphi=0$. We are not interested by such a contribution which does not play any role in the resonance phenomenon. In Eq. (11), terms like $\exp \left(i \lambda_{p}(k)(\varphi \pm m 2 \pi)\right)$ are surface wave contributions. A Regge pole lying in the first (resp. the third) quadrant of the CAM plane corresponds to a surface wave propagating counterclockwise (resp. clockwise) around the magnetic vortex and $\operatorname{Re} \lambda_{p}(k)$ represents its azimuthal propagation constant while $\operatorname{Im} \lambda_{p}(k)$ is its damping constant. In (11), we take into account the multiple circumnavigations around the magnetic vortex. Fig. 3 exhibits the distribution of Regge poles for a given wave number. The Regge poles in the first and third quadrants are not symmetrically distributed as a consequence of the breaking of time-reversal invariance. The Regge pole $\mathrm{RP} 0+$ is very close to the real axis in the complex $\lambda$-plane. It then corresponds to a surface wave which is slightly attenuate during its propagation and which contributes significantly to the scattering process and to the resonance mechanism. The Regge pole RP1+ is not so close to the real axis but it could have an appreciable influence on the resonance mechanism while the other Regge poles are too far to contribute so significantly.

Resonance phenomenons (and the resonant behavior of $f_{S}$ and therefore of $\sigma_{T}$ ) can now be understood in terms of surface waves. As $k$ varies, each Regge pole $\lambda_{p}(k)$ describes a trajectory (usually designed as a Regge trajectory [7]) in the CAM plane. When the quantity $\operatorname{Re} \lambda_{p}(k)$ 

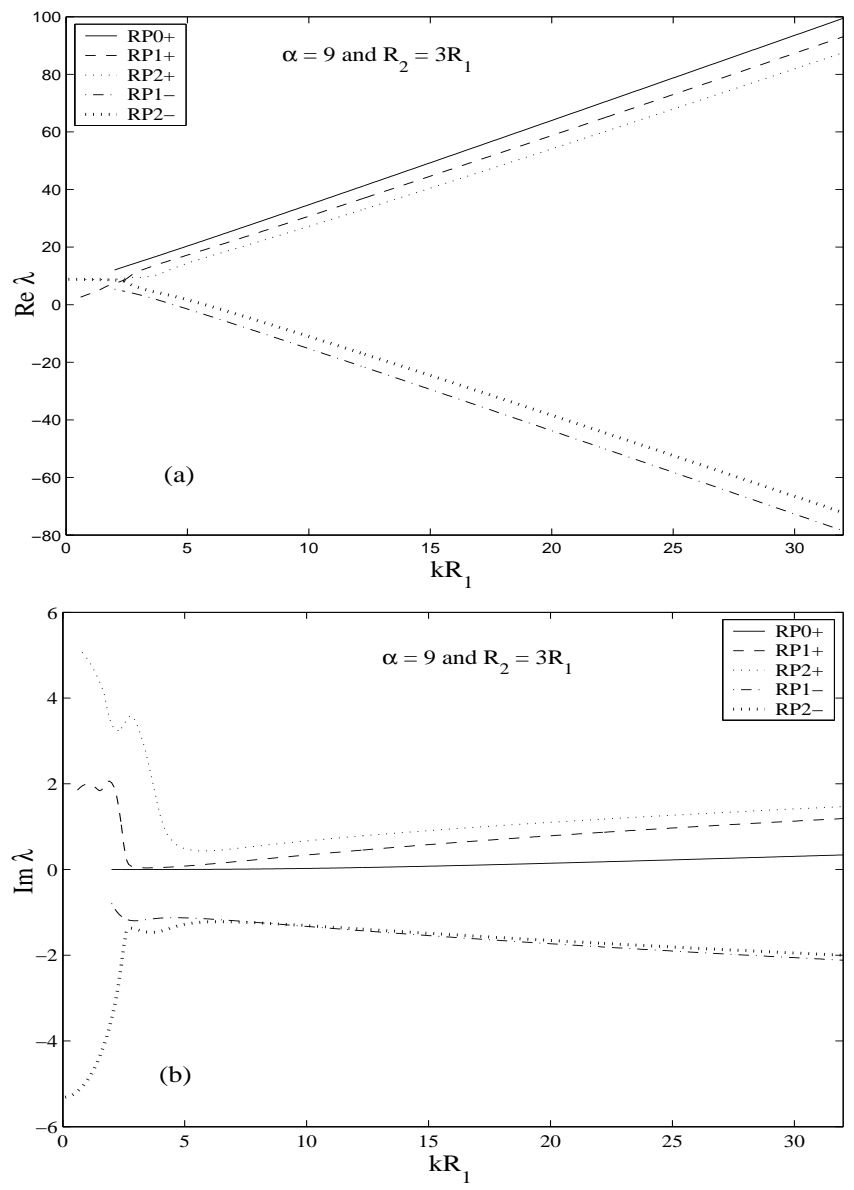

FIG. 4: Regge trajectories.

coincides with an integer, a resonance occurs. Indeed, it is produced by a constructive interference between the different components of the pth surface wave, each component corresponding to a different number of circumnavigations. Resonance wave numbers are therefore obtained from the Bohr-Sommerfeld quantization condition

$$
\operatorname{Re} \lambda_{p}\left(k_{\ell p}^{(o)}\right)=\ell \quad \text { for } \quad \ell \in \mathbf{Z}
$$

By assuming that $k$ is in the neighborhood of $k_{\ell p}^{(o)}$ and using $\operatorname{Re} \lambda_{p}(k) \gg \operatorname{Im} \lambda_{p}(k)$ (which can be numerically verified, except for very low frequencies), we can expand $\lambda_{p}(k)$ in a Taylor series about $k_{\ell p}^{(o)}$, and obtain

$$
\begin{aligned}
\lambda_{p}(k) \approx \ell+ & \left(\frac{d \operatorname{Re} \lambda_{p}(k)}{d k}\right)_{k=k_{\ell p}^{(o)}}\left(k-k_{\ell p}^{(o)}\right) \\
& +i \operatorname{Im} \lambda_{p}\left(k_{\ell p}^{(o)}\right) .
\end{aligned}
$$

Then, after summation over $m$ in (11), we find, by using (13), that $f_{S}(k, \varphi)$ presents a resonant behavior given by the Breit-Wigner formula (8) with

$$
\frac{\Gamma_{\ell p}}{2}=\left(\frac{\operatorname{Im} \lambda_{p}(k)}{d \operatorname{Re} \lambda_{p}(k) / d k}\right)_{k=k_{\ell p}^{(o)}} .
$$

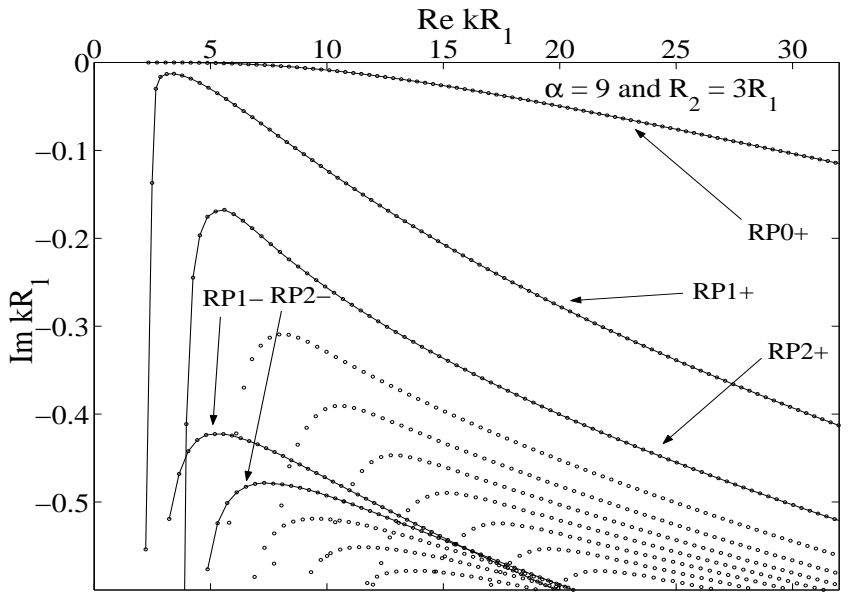

FIG. 5: Semiclassical resonances in the complex $k R_{1}$-plane.

Eqs. (12) and (14) are semiclassical formulas which permit us to determine the location of the resonances from Regge trajectories. Fig. 5 exhibits the resonance distribution obtained from the semiclassical formulas (12) and (14) by using the Regge trajectories numerically calculated (see Fig. 4). A comparison of Fig. 5 and Fig. 2 (or a numerical comparison between the exact and the semiclassical spectra) shows a very good agreement, except for low values of the wave number $k$. Furthermore, as indicated in Fig. 5 , the semiclassical theory permits us to classify the resonances in distinct families, each family being associated with one Regge pole and therefore to understand the meaning of the index $p$ introduced to denote the solutions of (7). In Fig. 6, a zoom in on Fig. 1 is realized and the peaks are associated with the semiclassical resonances linked to the Regge poles $\mathrm{RP} 0+$ and $\mathrm{RP} 1+$.

A deeper understanding of the scattering process as well as its dependence on the quantum flux parameter $\alpha$ can be obtained by solving perturbatively Eq. (10). Wait 12] presents a method in order to study acoustic whispering-gallery phenomena in circular cylinders. Such a method is equally valid, mutatis mutandis, for finding the solutions of (10). By using in (4) the appropriate asymptotic expansions [11] (Debye expansions and uniform expansions in terms of the Airy function) for Bessel functions, we can obtain an asymptotic approximation for the Regge poles $\lambda_{p}(k)$ :

$$
\begin{aligned}
& \lambda_{\mathrm{RP} 0+}(k)=k R_{2}+\alpha+u_{0}^{+}\left(k R_{2}\right)\left(\frac{k R_{2}}{2}\right)^{1 / 3}+\ldots \\
& \lambda_{\mathrm{RP} p \pm}(k)= \pm k R_{2}+\alpha \pm u_{p}^{ \pm}\left(k R_{2}\right)\left(\frac{k R_{2}}{2}\right)^{1 / 3}+\ldots
\end{aligned}
$$

with $p=1,2, \ldots$ Here $u_{0}^{+}\left(k R_{2}\right)$ and $u_{p}^{ \pm}\left(k R_{2}\right)$ are given 


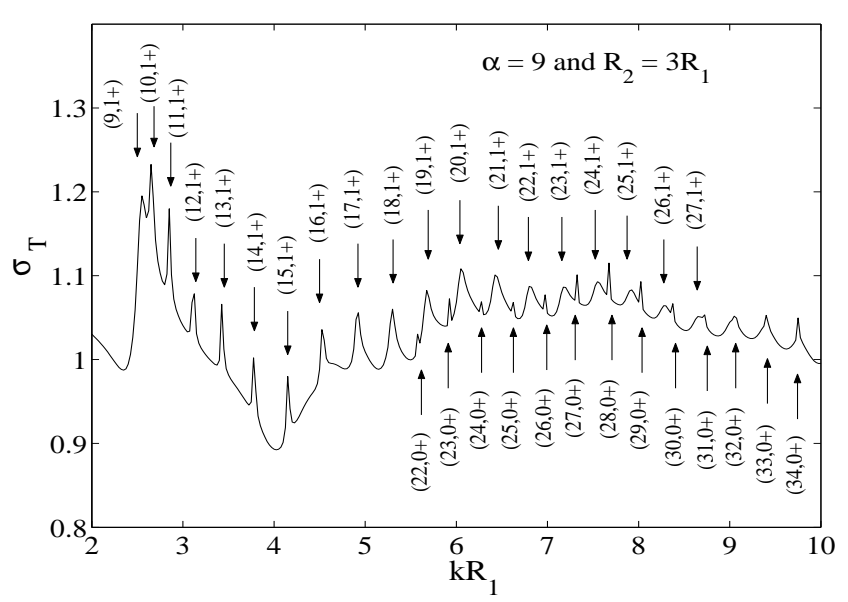

FIG. 6: Identification of resonances on the total cross section.

TABLE I: A comparison between the exact and the asymptotic values for Regge poles $\left(\alpha=9, R_{2}=3 R_{1}\right.$ and $\left.k R_{1}=18\right)$.

\begin{tabular}{ccc}
\hline \hline Regge poles & $\lambda_{p}$ exact & $\lambda_{p}$ semiclassical \\
\hline RP0+ & $+58.11+0.117 \mathrm{i}$ & $+57.69+0.305 \mathrm{i}$ \\
RP1+ & $+53.08+0.708 \mathrm{i}$ & $+53.21+0.470 \mathrm{i}$ \\
RP2+ & $+48.62+1.027 \mathrm{i}$ & $+48.52+0.840 \mathrm{i}$ \\
RP3+ & $+44.71+1.143 \mathrm{i}$ & $+44.50+0.866 \mathrm{i}$ \\
RP1- & $-38.02-1.658 \mathrm{i}$ & $-35.21-2.302 \mathrm{i}$ \\
RP2- & $-32.83-1.590 \mathrm{i}$ & $-30.52-1.739 \mathrm{i}$ \\
RP3- & $-28.62-1.552 \mathrm{i}$ & $-26.50-1.473 \mathrm{i}$ \\
\hline \hline
\end{tabular}

by

$$
\begin{aligned}
& u_{0}^{+}=t_{0}+i \frac{\tau_{0}{ }^{1 / 2}}{\left(-t_{0}\right)} \exp \left(-\frac{4}{3} \tau_{0}^{3 / 2}\right) \\
& u_{p}^{ \pm}=t_{p}+i \frac{\left(-\tau_{p \pm}\right)^{1 / 2}}{\left(-t_{p}\right)}
\end{aligned}
$$

where

$$
t_{0}=-\left(\frac{3 \pi}{4}\right)^{2 / 3}, t_{p}=-\left[\frac{3 \pi}{2}\left(p+\frac{1}{4}\right)\right]^{2 / 3},
$$

and

$$
\tau_{0}=t_{0}+\alpha\left(\frac{2}{k R_{2}}\right)^{1 / 3}, \tau_{p \pm}=t_{p} \pm \alpha\left(\frac{2}{k R_{2}}\right)^{1 / 3} .
$$

In Table II, we present some results for Regge poles. A comparison between the "exact" and the asymptotic values shows a rather good agreement. It could be possible to greatly improve the imaginary parts of the asymptotic results by taking into account higher orders of the perturbative series (15).

By replacing (15) in (11), it is obvious that all the surface waves are supported by the infinitely thin magnetic field shell localized at $\rho=R_{2}$. Furthermore, because of the term $\alpha$ which appears in (15), surface wave propagating around the magnetic vortex acquire a geometrical phase $\alpha \varphi \pm \alpha m 2 \pi$ (the + and - signs correspond respectively to surface waves propagating counterclockwise and clockwise) reminiscent of the AharonovBohm effect. Resonances are therefore associated with whispering-gallery modes mainly concentrated at the inside surface of the magnetic field discontinuity at $\rho=R_{2}$. Here we have encountered, in the context of quantum mechanics, the whispering-gallery phenomena described long time ago by Rayleigh 13, 14] in acoustics.

\section{CONCLUSION AND PERSPECTIVES}

To conclude, we would like first to comment on some aspects of our work and then to consider some possible directions which are suggested by our results:

- We have investigated the resonant properties of a rather special magnetic vortex for which the Schrödinger equation can be solved exactly in terms of Bessel functions. As a consequence, we have been able to perform the exact as well as the asymptotic calculations involved. We think that scattering by such a vortex cannot be considered as a thought experiment. Such a vortex could be realized and experimentally studied because, in the domain of the $\mathrm{AB}$ effect, experimentalists have developed ingenious techniques (see, for example, Ref. (1). At any rate, even if that is not possible, we think that resonant properties as well as surface waves could be experimentally observed from more general magnetic vortices.

- The CAM method could be naturally used in many other areas of the physics of vortices and in particular in order to study, from a semiclassical point of view, scattering by vortices in superfluids and Bose-Einstein condensates or in superconductors. However, it seems to us that our approach could be above all profitably extended in all the domains where analogs of the $\mathrm{AB}$ effect have been recently developed, extending the pioneering contribution of Berry et al 15] (see Refs. 16, 17, 18 for the acoustical AB effect, Refs. 19, 20, 21, 22 for the hydrodynamical $A B$ effect and Ref. 23 for the optical $A B$ effect). In the particular case of the scattering of ultrasonic waves by hydrodynamic vortices, the CAM method could provide new interpretations of sound and flow interactions in connection with the geometrical theory of diffraction.

- Finally, applications in the domain of electronics and mesoscopic physics could be envisaged and the resonant magnetic vortex used to modify the electron transport behavior. Surface waves as well as the associated resonances could not only contribute significantly to conductance oscillations, but also lead to new effects.

\section{Acknowledgments}

We thank Remy Berthet for fruitful discussions. 
[1] T. Ando, Y. Arakawa, K. Furuya, S. Komiyama, and H. Nakashima, Mesoscopic Physics and Electronics (Springer-Verlag, Berlin, 1998).

[2] Y. Aharonov and D. Bohm, Phys. Rev. 115, 485 (1959).

[3] S. Olariu and I. I. Popescu, Rev. Mod. Phys. 57, 339 (1985).

[4] M. Peshkin and A. Tonomura, The Aharonov-Bohm Effect, Lecture Notes in Physics, Vol. 340 (Springer-Verlag, Berlin, 1989).

[5] J. Q. Liang, Phys. Rev. D 32, 1014 (1985).

[6] G. N. Watson, Proc. Roy. Soc. London A 100, 83 (1918).

[7] R. G. Newton, Scattering Theory of Waves and Particles (Springer-Verlag, New-York, 1982), 2nd ed.

[8] H. M. Nussenzveig, Diffraction Effects in Semiclassical Scattering (Cambridge University Press, Cambridge, 1992).

[9] M. Berry, Eur. J. Phys. 1, 240 (1980).

[10] N. F. Mott and H. S. W. Massey, The Theory of Atomic Collisions (Oxford University Press, Oxford, 1965).

[11] M. Abramowitz and I. A. Stegun, Handbook of Mathematical Functions (Dover, New-York, 1965).

[12] J. R. Wait, Can. J. Phys. 45, 1861 (1967).
[13] J. W. S. Rayleigh, The Theory of Sound reprinted by Dover in 1945 (Dover, New-York, 1887).

[14] J. W. S. Rayleigh, Phil. Mag. 20, 1001 (1910).

[15] M. Berry, R. Chambers, M. Large, C. Upstill, and J. Walmsley, Eur. J. Phys. 1, 154 (1980).

[16] P. Roux, J. de Rosny, M. Tanter, and M. Fink, Phys. Rev. Lett. 79, 3170 (1997).

[17] S. Manneville, P. Roux, M. Tanter, A. Maurel, M. Fink, F. Bottausci, and P. Petitjeans, Phys. Rev. E 63, 036607 (2001).

[18] R. Berthet, Interaction son-écoulement, Ph.D. Thesis (Ecole Normale Supérieure de Lyon, 2001).

[19] F. Vivanco, F. Melo, C. Coste, and F. Lund, Phys. Rev. Lett. 83, 1966 (1999).

[20] C. Coste, F. Lund, and M. Umeki, Phys. Rev. E 60, 4908 (1999).

[21] C. Coste and F. Lund, Phys. Rev. E 60, 4917 (1999).

[22] R. Bernal, C. Coste, F. Lund, and F. Melo, Phys. Rev. Lett. 89, 034501 (2002).

[23] D. Neshev, A. Nepomnyashchy, and Y. S. Kivshar, Phys. Rev. Lett. 87, 043901 (2001). 